\title{
Zonation patterns of benthic communities in an upwelling area from the western Mediterranean (La Herradura, Alboran Sea)*
}

\author{
EMMA CEBRIÁN and ENRIC BALLESTEROS \\ Centre d'Estudis Avançats de Blanes (CSIC), C. Santa Bàrbara s/n. 17300 Blanes, Girona. Spain.
}

\begin{abstract}
Species composition and distribution of marine benthic communities from La Herradura (Alboran Sea, western Mediterranean) are described to characterise its rocky and sedimentary bottoms bionomically. Rocky bottoms were studied by means of several underwater transects and soft bottoms with fixed stations along a bathymetric gradient. The study of the floristic and faunistic composition of the rocky benthic communities highlights depth as the main axis of variation. Factorial Correspondence Analysis segregates deep-water communities below $25 \mathrm{~m}$ depth (circalittoral communities) from shallower communities (axis I), and communities thriving between 5 and $25 \mathrm{~m}$ depth (lower infralittoral communities) from communities thriving close to the surface (shallow infralittoral communities) (axis II). The study of the sedimentary bottoms also suggests that depth, together with physical sedimentary properties, is the main axis of variation in species distribution. Floristic and faunistic records show the particular composition of La Herradura benthic communities, compared to Mediterranean and Atlantic ones. Mixing of Mediterranean and Atlantic waters, together with deep water upwelling episodes typical of this area, probably determine the peculiar composition of the benthic communities.
\end{abstract}

Key words: zonation patterns, benthic communities, rocky and sedimentary bottoms, species distribution, Alboran Sea.

RESUMEN: PATRONES DE ZONACIÓN EN LAS COMUNIDADES BENTÓNICAS UNA ZONA DE AFLORAMIENTO DEL MEDITERRÁNEO OcCidental (La HerRadura, MAR de Alborán). - Se describen la composición y distribución de las comunidades bentónicas de La Herradura (Mar de Alborán, Mediterráneo Occidental) con el fin de caracterizar bionómicamente sus fondos rocosos y sedimentarios. Los fondos rocosos se estudiaron mediante transectos submarinos y los fondos sedimentarios mediante el muestreo de puntos fijos a lo largo de un gradiente batimétrico. La composición florística y faunística permite discernir la profundidad como eje de variación principal en los fondos rocosos. Tras aplicar un análisis factorial de correspondencias a los inventarios efectuados se observa que el primer eje segrega las comunidades situadas por debajo de los 25 metros (comunidades circalitorales) de las más superficiales (comunidades infralitorales), mientras que el segundo eje principal separa las comunidades situadas entre 5 y 25 metros (comunidades de la zona infralitoral inferior) de las situadas por encima de los 5 metros de profundidad (comunidades de la zona infralitoral superior). El estudio del fondo sedimentario refleja también la profundidad como factor predominante en la composición de las comunidades y, asociado a él, el tipo de sedimento encontrado. Todos los inventarios evidencian también la particular composición de los fondos estudiados, muy distinta a la del resto del Mediterráneo y diferente también a la del vecino Atlántico. La mezcla de aguas atlánticas y mediterráneas junto a los episodios de afloramiento de aguas profundas propios de esta zona son probablemente los factores determinantes de esta bionomía tan particular.

Palabras clave: patrones de zonación, comunidades bentónicas, fondos rocosos y sedimentarios, distribución de especies, Mar de Alborán. 


\section{INTRODUCTION}

The Alboran Sea is located at the westernmost part of the Mediterranean and has its own physical and biological traits (Gil, 1988). Surface Atlantic waters enter the Mediterranean through the Strait of Gibraltar and reach the Alboran Sea poorly mixed with Mediterranean waters (Cano, 1978). Furthermore, Atlantic waters usually describe an anticyclonic gyre that generates an upwelling of deep waters along the coasts of Málaga and Granada (southern Spain; Lanoix, 1974; Parrilla and Kinder, 1987; Minas et al., 1991; Tintoré et al., 1991). Deep water upwellings are enhanced by the strong western winds that usually blow in this area (Rodríguez, 1990). The combination of all these hydrological features together with the pronounced biogeographical differences between the Atlantic Ocean and the Mediterranean Sea (Conde, 1989; Pérès, 1985; González and Conde, 1993; Maldonado and Uriz, 1995; Cebrian et al. 2000) should affect benthic communities and their zonation patterns.

Although submerged vegetation patterns have been studied in several localities of the Alboran Sea (Giaccone, 1972; González, 1994), species composition of some circalittoral communities has been described (Templado et al., 1986; Templado et al., 1993; Maldonado, 1992, 1993), and the knowledge of the main invertebrates thriving in its waters is compiled in Ocaña et al. (2000), there are no available data on macroalgae and macrofauna species composition and distribution of communities along depth gradients in shallow $(0-50 \mathrm{~m})$ waters, with the exception of two transects described in Cebrian et al. (2000).
In the present study we describe the species composition and the zonation patterns of benthic communities thriving in the rocky and sedimentary bottoms of the village of La Herradura, whose coastal waters are regularly affected by deep water upwellings (Lanoix, 1974; Rodríguez, 1990; Templado et al., 1993), and we compare the structure of the studied communities and their spatial distribution with similar studies performed in other western Mediterranean localities.

\section{MATERIALS AND METHODS}

La Herradura ( $\left.36^{\circ} 44^{\prime} \mathrm{N}, 3^{\circ} 45^{\prime} \mathrm{W}\right)$ (Fig. 1), was selected amongst other localities of the southern coast of Spain due to its geographical position in front of an upwelling area, its rather steep sea bottom slopes which are responsible for a high landscape diversity, the presence of deep rocky bottoms close to the coast, and its diving facilities. Sampling was performed with SCUBA diving techniques (Zabala et al., 1982) between 18 June and 6 July 1997.

Sampling stations were selected covering different substrates, ranges of wave exposure and orientation. Identification of communities was performed following a transect perpendicular to the shore at every sampling station.

Transects on rocky bottoms were performed by two divers. In the first stage (descent) the topographic and bathymetric characteristics of the transect were noted, from the surface to the deeper zone (Table 1). Different communities were distinguished

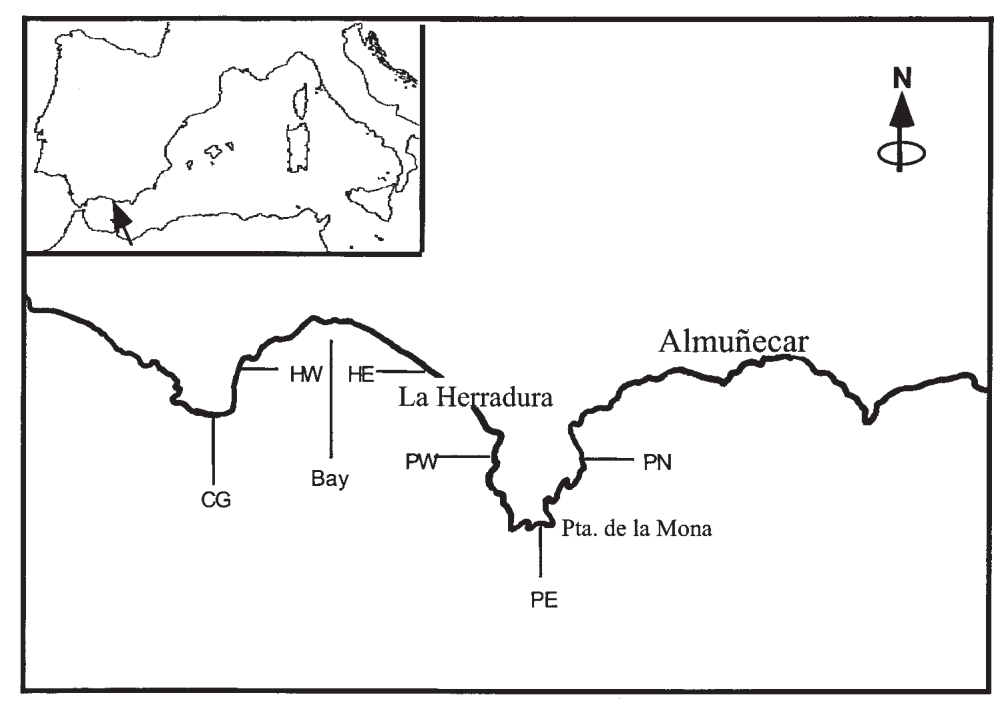

FIG. 1. - Location of the transects in La Herradura. 
TABLE 1. - Code, depth, orientation and kind of substrate for each sample.

\begin{tabular}{|c|c|c|c|c|c|}
\hline Code & Depth (m) & Transect & Slope & Orientation & Substratum \\
\hline $1 \mathrm{CG}$ & $>-24$ & Cerro Gordo & gently slope & south-east & small boulders \\
\hline $2 \mathrm{CG}$ & $23-17$ & & subhorizontal & & rocky \\
\hline $3 \mathrm{CG}$ & $10-17$ & & vertical cliff & & rocky \\
\hline $4 \mathrm{CG}$ & $0-10$ & & vertical cliff & & rocky \\
\hline 1HW & $>-15$ & La Herradura (west) & gently slope & east & muddy bottom \\
\hline $2 \mathrm{HW}$ & $15-10$ & & gently slope & & large boulders \\
\hline $3 \mathrm{HW}$ & -11 & & gently slope & & crevice \\
\hline $4 \mathrm{HW}$ & $10-5$ & & gently slope & & large boulders \\
\hline $5 \mathrm{HW}$ & $5-1,5$ & & steep slope & & rocky \\
\hline $6 \mathrm{HW}$ & $1,5-0$ & & steep slope & & rocky \\
\hline $1 \mathrm{HE}$ & $12-20$ & La Herradura (east) & gently slope & west & small boulders \\
\hline $2 \mathrm{HE}$ & $10-12$ & & gently slope & & big boulders \\
\hline $3 \mathrm{HE}$ & $8-10$ & & steep slope & & big boulders \\
\hline $4 \mathrm{HE}$ & $2-8$ & & steep slope & & big boulders \\
\hline $5 \mathrm{HE}$ & $0-2$ & & steep slope & & rocky \\
\hline $1 \mathrm{PW}$ & $>-38$ & Punta de la Mona (west) & gently slope & south & detritic bottom \\
\hline $2 \mathrm{PW}$ & $23-28$ & & gently slope & & large boulders \\
\hline 3PW & $12-23$ & & gently slope & & large boulders \\
\hline 4PW & $6-12$ & & vertical cliff & & rocky bottom \\
\hline 5PW & $0-6$ & & vertical cliff & & rocky bottom \\
\hline $1 \mathrm{PE}$ & $>-47$ & Punta de la Mona (east) & gently slope & south & detritic bottom \\
\hline $2 \mathrm{PE}$ & $46-30$ & & steep slope & & rocky bottom \\
\hline 3PE & $20-30$ & & steep slope & & rocky bottom \\
\hline 4PE & $10-20$ & & steep slope & & rocky bottom \\
\hline $5 \mathrm{PE}$ & $5-10$ & & steep slope & & rocky bottom \\
\hline $6 \mathrm{PE}$ & $<-6$ & & steep slope & & rocky bottom \\
\hline $1 \mathrm{PN}$ & $>-14$ & Punta de la Mona (north) & gently slope & north east & detritic bottom \\
\hline $2 \mathrm{PN}$ & $10-14$ & & very steep & & rocky bottom \\
\hline 3PN & $8-10$ & & very steep & & rocky bottom \\
\hline $4 \mathrm{PN}$ & $4-8$ & & very steep & & rocky bottom \\
\hline $5 \mathrm{PN}$ & $0-4$ & & very steep & & rocky bottom \\
\hline
\end{tabular}

along the transect according to the dominant species. In the second stage (ascent) each community was carefully checked for ten minutes and its species composition and abundances were recorded according to a semi-quantitative index (Braun Blanquet, 1979). Qualitative samples of each predefined community were collected for identification of the organisms in the laboratory.

On sedimentary bottoms the sampling was performed at 5 different stations located along a transect situated in the middle of the bay. Dives were performed at depths of 10, 20, 30, 40 and 50 metres and species composition and abundance were recorded for ten minutes, with collection of samples for identification of the organims. Sediment samples were collected using two cores and two dredges (van Veen) at each sampling station. Dredges were obtained by ordinary methods below $50 \mathrm{~m}$ depth and characterised qualitatively by their species composition. Cores were of $12.5 \mathrm{~cm}$ diameter and $30 \mathrm{~cm}$ length. Small superficial sediment subsamples were collected from the core for granulometrical analysis. They were analysed using an LS particle size analyser at the Department of Geology of the University of Barcelona. The grain size distribution of the sediment was performed following Wentworth (1972) classification.

Data obtained from the transects were used to describe them and to draw precise sketches of benthic communities.

A factorial correspondence analysis (Legendre and Legendre, 1979; Ter Braak, 1987) was performed with the samples collected on rocky bottoms to show affinities and differences between samples. Mean percent abundance values obtained from the six rocky transects were used as data entries in a species-station raw matrix. Species present in only one or two samples were not considered in the analysis. Sample $3 \mathrm{HO}$ collected in a crevice was also omitted from the analysis due to its very peculiar species composition. The final analysed matrix included 30 points and 84 taxa.

\section{RESULTS}

Species composition and abundances for each sample are indicated in Table 2 (rocky transects) and in Table 3 (sedimentary transect) and are diagrammatically shown in Figures 2 to 5. 
TABLE 2. - Species composition and abundance in the communities distinguished along the six rocky transects, according to Braun Blanquet index. Key signs: (+) presence, (1) 1-10\% abundance, (2) 10-25\% abundance, (3) $25-50 \%$ abundance, (4) $50-75 \%$ abundance. *crevice. Ordination of species in the table follows criteria of abundance.

\begin{tabular}{|c|c|c|c|c|c|c|c|c|c|c|c|}
\hline & & & Cerro & Gord & & & $\mathrm{La} \mathrm{H}$ & erra & 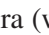 & vest) & \\
\hline & & $1 C G$ & $2 \mathrm{CG}$ & $3 C G$ & $4 \mathrm{CG}$ & $1 \mathrm{HW}$ & $2 \mathrm{HW}$ & & 4HV & $5 \mathrm{HW}$ & $6 \mathrm{HW}$ \\
\hline 1 & Cliona viridis & 2 & 2 & 1 & 1 & 2 & 1 & - & 1 & 1 & - \\
\hline 2 & Mesophyllum alternans & - & 2 & 3 & 1 & 2 & 2 & - & 2 & 1 & 2 \\
\hline 3 & Astroides calycularis & + & + & 1 & 1 & - & - & - & 1 & 2 & + \\
\hline 4 & Schizobrachiella sanguinea & - & 1 & 2 & 1 & - & - & - & 2 & 2 & - \\
\hline 5 & Crambe crambe & - & 2 & + & 1 & - & 1 & 1 & 1 & 1 & - \\
\hline 6 & Lithophyllum incrustans & - & - & + & 2 & - & 1 & - & 2 & 5 & 1 \\
\hline 7 & Aiptasia mutabilis & - & - & 1 & + & - & 1 & - & 2 & - & - \\
\hline 8 & Asparagopsis armata & + & 1 & 2 & 2 & - & - & - & $\overline{2}$ & - & 2 \\
\hline 9 & Halopteris filicina & 1 & 2 & - & - & 2 & 2 & - & - & - & - \\
\hline 10 & Aglaophenia sp. & 1 & 2 & - & - & 2 & 2 & - & 2 & 2 & 2 \\
\hline 11 & Arbacia lixula & - & - & 2 & 1 & - & - & - & 1 & $\overline{3}$ & + \\
\hline 12 & Peyssonnelia rosa-marina & - & - & 2 & 3 & - & 1 & - & 1 & + & - \\
\hline 13 & Aglaozonia sp. & - & - & - & 1 & - & - & - & 1 & 2 & 1 \\
\hline 14 & Paracentrotus lividus & - & - & - & 2 & - & - & - & 1 & 2 & + \\
\hline 15 & Plocamium cartilagineum & 1 & 1 & 1 & 1 & - & - & - & - & - & - \\
\hline 16 & Sphaerechinus granularis & & & + & 1 & - & - & - & 1 & 1 & - \\
\hline 17 & Aphanocladia stichidiosa & 1 & 2 & 1 & 2 & - & - & - & - & - & - \\
\hline 18 & Holothuria sanctori & - & 2 & 2 & 1 & - & - & - & 1 & + & - \\
\hline 19 & Myriapora truncata. & + & + & + & - & 1 & 1 & - & 1 & - & - \\
\hline 20 & Serpula vermicularis & - & + & - & - & - & - & - & + & - & + \\
\hline 21 & Chondrosia reniformis & - & 1 & - & - & - & 1 & 1 & - & - & - \\
\hline 22 & Dictyota dichotoma & + & + & - & - & 1 & 1 & - & 1 & - & + \\
\hline 23 & Salmacina dysteri & 1 & 1 & + & + & - & - & 2 & - & - & - \\
\hline 24 & Clathrina cerebrum & - & 1 & - & - & - & - & - & + & + & - \\
\hline 25 & Actinothoe sphyrodeta & - & - & - & 2 & - & - & - & + & 2 & 2 \\
\hline 26 & Dysidea avara & - & - & - & - & - & - & 1 & - & - & - \\
\hline 27 & Ircinia fasciculata & - & 2 & - & - & - & 1 & - & 2 & 1 & - \\
\hline 28 & Cliona celata & - & - & 1 & 2 & - & - & - & - & - & - \\
\hline & Holothuria forskali & - & + & - & - & - & + & + & + & - & - \\
\hline & Protula sp. & - & - & - & - & - & + & + & - & - & - \\
\hline 29 & Pseudodistoma crucigaster & - & - & - & - & - & - & - & - & - & - \\
\hline 30 & Alcyonium acaule & - & - & - & - & - & - & - & - & - & - \\
\hline 31 & Clavelina dellavalle $i$ & - & 2 & 2 & - & - & - & - & - & - & - \\
\hline 32 & Codium bursa & - & - & - & - & - & - & - & + & - & - \\
\hline 33 & Corynactis viridis & - & - & - & 1 & - & - & - & - & - & 2 \\
\hline 34 & Holothuria tubulosa & 1 & - & - & - & 2 & 2 & - & + & - & - \\
\hline & Marthasterias glacialis. & & - & - & + & - & - & - & + & + & - \\
\hline 35 & Rhodymenia ardissonei & 1 & 1 & - & - & - & - & - & - & - & - \\
\hline 36 & Corallina elongata & - & - & - & - & - & - & - & - & - & 3 \\
\hline 37 & Hemimycale columella & - & 1 & - & - & - & 1 & - & - & - & - \\
\hline 38 & Peyssonnelia sp. & - & - & - & + & - & 1 & - & - & - & - \\
\hline 39 & Phorbas fictitius & - & 1 & - & - & - & - & + & - & - & - \\
\hline & Schizomavella discoidea & - & 1 & - & - & 1 & 1 & + & - & - & - \\
\hline 40 & Amphiroa beauvoisii & - & - & - & - & - & - & - & - & - & 3 \\
\hline 41 & Axinella damicornis & - & + & - & - & - & - & 2 & - & - & - \\
\hline & Bryopsis plumosa & & - & - & - & - & - & - & - & - & - \\
\hline 42 & Cerianthus membranaceus & 1 & - & - & - & - & - & - & - & - & - \\
\hline & Chartella tenella & - & - & - & - & - & + & 3 & - & - & - \\
\hline & Clavularia crassa & - & - & + & - & - & - & - & 1 & - & - \\
\hline 43 & Falkenbergia rufolanosa & - & - & - & - & - & - & - & - & - & - \\
\hline & Ophidiaster ophidianus & - & - & - & - & - & - & - & - & - & - \\
\hline 44 & Pentapora fascialis & 1 & 2 & - & - & - & - & - & - & - & - \\
\hline & Peyssonnelia coriacea & - & - & - & - & - & - & - & - & - & - \\
\hline & Sarcotragus spinosula & - & + & - & - & - & - & - & - & - & - \\
\hline & Acrosorium venulosum & + & + & - & - & - & - & - & - & - & - \\
\hline & Adeonella calveti & - & - & - & - & - & + & 2 & - & - & - \\
\hline & Bonnemaisonia asparagoides & - & - & - & - & - & + & - & - & - & - \\
\hline & Clavelina nana & - & + & - & - & - & - & - & - & - & - \\
\hline & Colpomenia sinuosa & - & - & - & - & - & - & - & 1 & + & - \\
\hline 45 & Didemnит maculosum & - & - & - & - & - & - & - & - & - & - \\
\hline & Gelidium pusillum & - & - & - & - & - & - & - & - & - & - \\
\hline & Haliclona mediterranea & - & - & - & - & - & - & - & - & - & - \\
\hline & Oscarella lobularis & - & - & - & - & - & - & 1 & - & - & - \\
\hline 46 & Parazoanthus axinellae & - & - & - & - & - & - & - & - & - & - \\
\hline & Rynchozoon neapolitanum & - & - & - & - & - & - & - & - & - & - \\
\hline 47 & Actinia sp. & - & - & - & - & - & - & - & - & - & - \\
\hline & Bonellia viridis & - & - & - & - & + & + & - & - & + & - \\
\hline & Cliona smithii & - & - & - & - & - & - & - & - & - & - \\
\hline & Codium vermilara & - & - & - & - & - & - & - & - & - & - \\
\hline 48 & Dendrophyllia ramea & - & - & - & - & - & - & - & - & - & - \\
\hline & Diplosoma spongiforme & - & - & - & - & - & - & - & - & - & - \\
\hline & Echinaster sepositus & - & - & - & - & - & - & - & - & - & - \\
\hline & Eudendrium sp. & - & - & - & - & - & - & 2 & - & - & - \\
\hline & Hexadella racovitzai & - & - & - & - & - & - & - & - & - & - \\
\hline 49 & Ircinia variabilis & 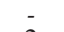 & - & - & - & - & - & - & - & - & - \\
\hline 50 & Leptogorgia sarmentosa & 2 & - & - & - & - & - & - & - & - & - \\
\hline & Padina pavonica & - & - & + & 1 & - & - & - & 1 & - & - \\
\hline & Paraerythropodium coralloides & - & - & - & - & - & - & - & - & - & - \\
\hline & Petrosia ficiformis & - & - & - & - & - & - & - & - & - & - \\
\hline & Peyssonnelia bornetii & - & - & + & - & - & + & - & - & - & - \\
\hline & Phallusia fumigata .. & - & - & - & - & - & - & - & 1 & + & - \\
\hline & Phyllangia mouchezii & - & - & - & - & - & - & - & - & - & - \\
\hline 51 & Rynchozoon sp. & - & - & - & - & - & - & - & - & - & - \\
\hline & Sabella spallanzani & - & - & - & + & + & + & - & - & - & - \\
\hline & Turbicellepora avicularis & - & - & - & - & - & - & - & - & - & - \\
\hline
\end{tabular}




\begin{tabular}{|c|c|c|c|c|c|c|c|c|c|}
\hline & & Cerro & Gord & & & & rra & $\mathrm{ra}($ & \\
\hline & $1 \mathrm{CG}$ & $2 \mathrm{CG}$ & $3 \mathrm{CG}$ & $4 \mathrm{CG}$ & $1 \mathrm{HW}$ & $2 \mathrm{HV}$ & HW & IHY & $5 \mathrm{HW}$ \\
\hline Aglaophenia pluma & - & - & - & - & - & - & - & - & - \\
\hline Axinella polypoides & - & - & - & - & - & - & - & - & - \\
\hline Balanophyllia regia & - & - & - & - & - & - & - & - & - \\
\hline Balanus perforatus & - & - & - & - & - & - & - & - & 1 \\
\hline Balliella cladoderma & - & - & - & - & - & + & - & - & - \\
\hline Botryocladia sp. & + & + & - & - & - & - & - & - & - \\
\hline Celleporina sp. & - & 1 & - & - & - & - & - & - & - \\
\hline Ceramium flaccidum & - & - & - & - & - & - & - & - & - \\
\hline Clathrina clathrus & - & - & - & - & - & - & - & - & - \\
\hline Clavelina lepadiformis & - & - & + & + & - & - & - & - & - \\
\hline Codium effusum & - & - & - & + & + & - & - & - & - \\
\hline Algal complex & - & - & - & - & - & - & - & - & - \\
\hline Contarinia squamariae & - & - & - & - & - & - & - & - & - \\
\hline Dictyonella incisa & - & - & - & - & - & - & - & - & - \\
\hline Didemnит pseudofulgens & - & - & - & - & - & - & - & - & - \\
\hline Didemnum sp. & - & - & - & - & - & - & - & - & - \\
\hline Erythroglossum sandrianum & - & - & - & - & - & - & - & - & - \\
\hline Eunicella verrucosa & + & - & - & - & - & - & - & - & - \\
\hline Eurypon sp. & - & 1 & - & - & - & - & 1 & - & - \\
\hline Ircinia oros & - & + & - & - & - & - & - & - & - \\
\hline Jania corniculata & - & - & - & - & - & - & - & - & - \\
\hline Jania rubens & - & - & - & - & - & - & - & - & - \\
\hline Leptopsammia pruvotii & - & - & - & - & - & - & - & - & - \\
\hline Lissoclinum perforatum & - & - & - & - & - & - & - & - & - \\
\hline Lomentaria ercegovicii & - & - & - & - & - & - & - & - & - \\
\hline Obelia bidentata & - & - & - & - & - & - & - & - & - \\
\hline Pleraplysilla spinifera & - & - & - & - & - & - & - & - & - \\
\hline Polysyncraton sp. & - & - & - & - & - & - & - & - & - \\
\hline Porella cervicornis & - & - & - & - & - & - & - & - & - \\
\hline Reptadeonella violacea & - & - & - & + & - & - & - & - & - \\
\hline Rynchozoon sp II. & - & - & - & - & - & - & - & - & - \\
\hline Savygniella lafontii & - & 1 & - & - & - & - & - & - & - \\
\hline Turbicellepora magnicostata & - & - & - & - & - & - & - & - & - \\
\hline Valonia macrophysa & - & - & - & + & - & - & - & - & - \\
\hline Acanthella acuta & - & - & - & - & - & - & - & - & - \\
\hline Aglaophenia acacia & - & - & - & - & - & - & - & - & - \\
\hline Aglaophenia kincherpaueri & - & - & - & - & - & - & - & - & - \\
\hline Aglaothamnion tripinnatum & - & - & - & - & - & - & - & - & - \\
\hline Alicia mirabilis & - & - & - & - & - & - & - & - & - \\
\hline Amphiroa cryptarthrodia & - & - & - & - & - & - & - & - & - \\
\hline Aplysina cavernicola & - & - & - & - & - & - & - & - & - \\
\hline Aplysilla sulphurea & - & - & - & - & - & - & - & - & - \\
\hline Bolma rugosa. & 1 & - & - & - & - & - & - & - & - \\
\hline Caberea boryi & - & - & - & - & - & - & - & - & - \\
\hline Calliostoma zizyphinum & - & - & - & - & - & - & - & - & - \\
\hline Celleporina caminata & - & - & - & - & - & - & - & - & - \\
\hline Centrostephanus longispinus & - & - & - & - & - & - & - & - & - \\
\hline Cereus pedunculatus & - & - & - & - & - & - & - & - & - \\
\hline Corallina granifera & - & - & - & - & - & - & - & - & - \\
\hline Cornularia cornucopiae & - & - & + & - & - & - & - & - & - \\
\hline Crella elegans & - & - & - & - & - & - & - & - & - \\
\hline Cutleria adspersa & - & - & - & - & - & - & - & - & - \\
\hline Cutleria monoica & - & - & - & - & - & + & - & - & - \\
\hline Dardanus callidus & - & - & - & - & - & - & - & - & - \\
\hline Dentiporella sardonica & - & - & - & - & - & - & - & - & - \\
\hline Dictyonella pelligera & - & - & - & - & - & - & - & - & - \\
\hline Didemnum cf. commune & - & - & - & - & - & - & - & - & - \\
\hline Didemnum coriaceum & - & - & - & - & - & - & - & - & - \\
\hline Epizoanthus arenaceus & - & - & - & - & - & - & - & - & - \\
\hline Eupolymnia sp. & - & - & - & - & - & - & - & - & - \\
\hline Gelidium latifolium & - & - & - & - & - & - & - & - & - \\
\hline Hacelia attenuata & - & - & - & - & - & - & - & - & - \\
\hline Haraldia lenormandii & - & - & - & - & - & - & - & - & - \\
\hline Holothuria sp. & - & - & + & - & - & - & - & - & - \\
\hline Cacospongia scalaris & - & - & - & - & - & - & - & - & - \\
\hline Kallymenia sp. & - & - & - & - & - & - & - & - & - \\
\hline Oscarella sp. & - & - & - & - & - & - & - & - & - \\
\hline Paramuricea clavata & - & - & - & - & - & - & - & - & - \\
\hline Parasmittina tropica & - & - & - & - & - & - & - & - & - \\
\hline Peyssonnelia magna & - & - & - & + & - & - & - & - & - \\
\hline Peyssonnelia rubra & - & - & - & - & - & - & - & - & - \\
\hline Phorbas tenacior & - & - & - & - & - & - & - & - & - \\
\hline Platoma cyclocolpa & - & - & - & - & - & - & - & - & - \\
\hline Unidentified Polychaeta & - & - & - & - & - & - & - & - & - \\
\hline Polycyathus muellerae & - & - & - & - & - & - & - & - & - \\
\hline Polysiphonia scopulorum & - & - & - & - & - & - & - & - & - \\
\hline Polysiphonia sp. & - & - & - & - & - & - & - & - & - \\
\hline Pterothamnion plumula & - & - & - & + & - & - & - & - & - \\
\hline Reniera sp. & - & - & - & - & - & - & - & - & - \\
\hline Schizomavella cuspidata & - & - & - & - & - & - & - & - & - \\
\hline Scinaia sp. & - & + & - & - & - & - & - & - & - \\
\hline Serpulorbis arenarius & - & - & - & - & - & - & - & - & - \\
\hline Stephanoscyphus mirabilis & - & - & - & - & - & - & - & - & - \\
\hline Turbicellepora plana & - & - & - & - & - & - & - & - & - \\
\hline Ulosa stuposa & - & - & - & - & - & - & - & - & - \\
\hline Veretillum cynomorium & - & - & - & - & - & - & - & - & - \\
\hline Walkeria uva & - & - & - & - & - & - & - & - & - \\
\hline
\end{tabular}


TABLE 2 (Cont.). - Species composition and abundance in the communities distinguished along the six rocky transects, according to Braun Blanquet index. Key signs: (+) presence, (1) 1-10\% abundance, (2) 10-25\% abundance, (3) $25-50 \%$ abundance, (4) $50-75 \%$ abundance. Ordination of species in the table follows criteria of abundance.

\begin{tabular}{|c|c|c|c|c|c|c|c|c|c|c|c|c|}
\hline & & Pta. la & Mon & a (we & & & Pta & la M & ona $(e$ & ast) & & \\
\hline & $1 \mathrm{PW}$ & $2 \mathrm{PW}$ & 3PW & $4 \mathrm{PW}$ & $5 \mathrm{PW}$ & $1 \mathrm{PE}$ & $2 \mathrm{PE}$ & $3 \mathrm{PE}$ & $4 \mathrm{PE}$ & $5 \mathrm{PE}$ & $6 \mathrm{PE}$ & $1 \mathrm{PN}$ \\
\hline Cliona viridis & 2 & 3 & 1 & 1 & 1 & 2 & 2 & 1 & 1 & 1 & + & 3 \\
\hline Mesophyllum alternans & 1 & 2 & 2 & 1 & 2 & - & - & - & 3 & 2 & 1 & 3 \\
\hline Astroides calycularis & - & 1 & 2 & 1 & 1 & - & - & 1 & 1 & 3 & 3 & + \\
\hline Schizobrachiella sanguinea & - & 1 & 1 & 1 & 2 & + & - & 1 & 2 & + & 2 & + \\
\hline Crambe crambe & - & 1 & - & - & - & - & - & 1 & 1 & + & 1 & 1 \\
\hline Lithophyllum incrustans & - & - & + & 3 & 2 & - & - & - & 1 & 2 & + & 3 \\
\hline Aiptasia mutabilis & - & - & 1 & 1 & 1 & - & - & - & 1 & + & 1 & 1 \\
\hline Asparagopsis armata & - & - & 1 & 2 & 2 & - & - & - & 2 & 2 & + & 1 \\
\hline Halopteris filicina & 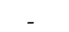 & 1 & 2 & + & - & - & + & 2 & 2 & + & + & 3 \\
\hline Aglaophenia sp. & 2 & 2 & - & - & - & 2 & 2 & 2 & - & - & & 1 \\
\hline Arbacia lixula & - & - & - & 1 & 1 & - & - & - & - & 1 & 1 & 1 \\
\hline Peyssonnelia rosa-marina & - & - & - & - & 2 & - & - & - & - & 1 & + & 3 \\
\hline Aglaozonia sp. & - & - & - & - & 1 & - & - & - & - & 1 & 1 & 2 \\
\hline Paracentrotus lividus & - & - & 1 & 2 & 1 & - & - & - & - & 1 & 1 & - \\
\hline Plocamium cartilagineum & - & - & & 2 & 1 & - & - & 1 & 1 & + & + & - \\
\hline Sphaerechinus granularis & - & - & 1 & 1 & - & - & - & - & 1 & - & - & - \\
\hline Aphanocladia stichidiosa & - & - & 2 & 2 & 2 & - & + & 1 & - & + & - & - \\
\hline Holothuria sanctori & - & - & 1 & - & - & - & - & 1 & 1 & 1 & - & - \\
\hline Myriapora truncata & - & - & 1 & - & - & - & - & 1 & + & - & - & - \\
\hline Serpula vermicularis & - & - & 1 & - & - & - & + & + & \pm & - & + & - \\
\hline Chondrosia reniformis & - & + & - & - & - & - & 1 & 1 & - & - & 1 & - \\
\hline Dictyota dichotoma & - & & - & - & - & - & - & - & - & - & - & 2 \\
\hline Salmacina dysteri & 1 & 3 & - & - & - & + & 2 & 1 & - & - & - & - \\
\hline Clathrina cerebrum & - & - & - & + & 1 & - & - & - & - & - & + & - \\
\hline Actinothoe sphyrodeta & - & - & + & 1 & 3 & - & - & - & - & 1 & - & - \\
\hline Dysidea avara & - & + & 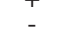 & 1 & - & + & 1 & 1 & + & + & 1 & - \\
\hline Ircinia fasciculata & - & - & - & - & - & - & - & - & - & - & - & - \\
\hline Cliona celata & - & - & - & 2 & + & - & - & - & 1 & 1 & + & - \\
\hline Holothuria forskali & - & - & 1 & - & - & - & - & - & - & - & - & - \\
\hline Protula sp. & - & - & - & - & - & - & - & - & - & - & + & - \\
\hline Pseudodistoma crucigaster & - & - & 2 & 1 & + & - & - & 1 & 1 & - & T- & - \\
\hline Alcyonium acaule & - & 2 & 1 & + & T- & - & 2 & 1 & - & - & - & - \\
\hline Clavelina dellavallei & 1 & 2 & - & - & - & - & 2 & 1 & 2 & - & - & - \\
\hline Codium bursa & - & - & - & 1 & 1 & - & - & - & 1 & - & + & - \\
\hline Corynactis viridis & - & - & - & - & - & - & - & - & - & + & + & - \\
\hline Holothuria tubulosa & - & - & - & - & - & - & - & 2 & - & - & - & 2 \\
\hline Marthasterias glacialis & + & + & - & - & - & - & - & - & + & - & + & - \\
\hline Rhodymenia ardissonei & T- & - & - & - & - & - & + & - & - & + & - & - \\
\hline Corallina elongata & - & - & - & + & 1 & - & - & - & - & - & - & - \\
\hline Hemimycale columella & + & + & - & - & - & - & 1 & 1 & - & - & - & - \\
\hline Peyssonnelia sp. & - & - & - & - & - & - & - & - & - & - & - & 1 \\
\hline Phorbas fictitius & - & + & - & - & - & - & - & - & - & - & 1 & - \\
\hline Schizomavella discoidea & - & - & - & - & - & + & - & - & 1 & - & 1 & 1 \\
\hline Amphiroa beauvoisii & - & - & - & - & + & - & - & - & 1 & - & - & - \\
\hline Axinella damicornis & - & - & - & - & - & - & 2 & 1 & - & - & - & - \\
\hline Bryopsis plumosa & - & - & - & - & - & - & - & - & - & - & 1 & - \\
\hline Cerianthus membranaceus & 1 & 2 & - & - & - & - & 2 & 1 & - & - & - & - \\
\hline Chartella tenella & - & - & - & - & - & - & - & + & + & - & - & - \\
\hline Clavularia crassa & - & - & - & - & - & - & - & - & - & - & - & - \\
\hline Falkenbergia rufolanosa & - & - & - & - & 1 & - & + & - & - & - & - & - \\
\hline Ophidiaster ophidianus & - & - & + & + & - & - & - & - & - & - & + & - \\
\hline Pentapora fascialis & - & 2,3 & - & - & - & - & 2 & 2 & 1 & - & - & - \\
\hline Peyssonnelia coriacea & - & - & - & - & - & - & + & - & + & + & 1 & - \\
\hline Sarcotragus spinosula & - & - & - & - & - & - & - & - & T- & - & 1 & - \\
\hline Acrosorium venulosum & - & - & - & - & - & - & + & - & - & - & - & - \\
\hline Adeonella calvet $i$ & - & - & - & - & - & + & - & - & - & - & - & - \\
\hline Bonnemaisonia asparagoides & - & - & - & - & - & - & + & + & - & - & - & + \\
\hline Clavelina nana & - & - & - & - & - & - & - & 1 & 1 & - & + & - \\
\hline Colpomenia sinuosa & - & - & - & - & 1 & - & - & - & - & - & - & - \\
\hline Didemnum maculosum & 1 & 2 & - & - & - & 1 & - & - & - & - & 1 & - \\
\hline Gelidium pusillum & 1 & - & - & - & - & 1 & - & - & - & - & + & - \\
\hline Haliclona mediterranea & - & - & - & - & - & - & - & - & - & - & + & - \\
\hline Oscarella lobularis & - & - & - & - & - & - & - & - & - & - & 1 & - \\
\hline Parazoanthus axinellae & - & + & - & - & - & - & 2 & 1 & - & - & - & - \\
\hline Rynchozoon neapolitanum & - & - & - & - & - & - & - & - & - & + & - & + \\
\hline Actinia sp. & - & - & - & 2 & 2 & - & - & - & - & 2 & - & - \\
\hline Bonellia viridis & - & - & - & - & - & - & - & - & - & - & - & - \\
\hline Cliona smithii & - & - & - & - & - & - & - & - & - & - & - & - \\
\hline Codium vermilara & - & - & - & - & - & 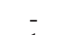 & - & - & - & + & + & - \\
\hline Dendrophyllia ramea & - & 1 & - & - & - & 1 & 2 & - & - & - & - & - \\
\hline Diplosoma spongiforme & - & - & - & - & - & - & - & - & + & - & + & - \\
\hline Echinaster sepositus & - & - & - & - & - & - & - & + & + & - & + & - \\
\hline Eudendrium sp. & - & - & - & - & - & - & - & - & - & - & - & - \\
\hline Hexadella racovitzai & - & - & - & - & - & - & 1 & 1 & - & - & - & - \\
\hline Ircinia variabilis & 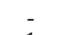 & - & - & - & - & - & - & - & - & - & - & - \\
\hline Leptogorgia sarmentosa & 1 & - & - & - & - & - & 1 & - & - & - & - & - \\
\hline Padina pavonica & - & - & - & - & - & - & 1 & - & - & - & - & - \\
\hline Paraerythropodium coralloide & es - & - & - & - & - & - & - & - & - & - & 1 & - \\
\hline Petrosia ficiformis & - & + & - & - & - & - & 1 & 1 & - & - & - & - \\
\hline Peyssonnelia bornetii & - & - & - & - & - & - & - & - & - & - & - & - \\
\hline Phallusia fumigata & . & - & - & - & - & - & - & - & - & - & - & - \\
\hline Phyllangia mouchezii & + & 1 & - & - & - & - & + & - & - & - & - & - \\
\hline Rynchozoon sp. & - & - & - & - & - & - & - & - & - & - & + & - \\
\hline Sabella spallanzani & i & - & - & - & - & $\dot{y}$ & - & - & - & - & - & - \\
\hline
\end{tabular}




\begin{tabular}{|c|c|c|c|c|c|c|c|c|c|}
\hline & & Pta. la & Mon & $\mathrm{a}$ (wes & & & & la M & ona $(\mathrm{e}$ \\
\hline & $1 \mathrm{PW}$ & 2PW & 3PW & $4 \mathrm{PW}$ & $5 \mathrm{PW}$ & 1PE & $2 \mathrm{PE}$ & $3 \mathrm{PE}$ & 4PE \\
\hline Aglaophenia pluma & 2 & 1 & - & - & - & - & - & - & - \\
\hline Axinella polypoides & - & + & - & - & - & - & 1 & - & - \\
\hline Balanophyllia regia & - & - & - & - & - & - & - & - & - \\
\hline Balanus perforatus & - & - & - & - & - & - & - & - & - \\
\hline Balliella cladoderma & - & - & - & - & - & - & - & - & - \\
\hline Botryocladia sp. & - & - & - & - & - & - & - & - & - \\
\hline Celleporina sp. & - & + & - & - & - & - & - & - & - \\
\hline Ceramium flaccidum & - & - & - & - & - & - & - & - & - \\
\hline Clathrina clathrus & - & - & - & - & - & - & - & - & - \\
\hline Clavelina lepadiformis & - & - & - & - & - & - & - & - & - \\
\hline Codium effusum & - & - & - & - & - & - & - & - & - \\
\hline Algal complex & - & - & - & - & - & - & - & - & - \\
\hline Contarinia squamariae & - & - & - & - & - & - & - & - & - \\
\hline Dictyonella incisa & - & - & - & - & - & - & + & + & - \\
\hline Didemnum pseudofulgens & - & - & - & - & - & - & - & - & - \\
\hline Didemnum sp. & - & - & - & - & - & - & - & - & - \\
\hline Erythroglossum sandrianum & - & 1 & - & - & - & - & + & - & - \\
\hline Eunicella verrucosa & - & - & - & - & - & + & - & - & - \\
\hline Eurypon sp. & - & - & - & - & - & - & - & - & - \\
\hline Ircinia oros & - & - & - & - & - & - & + & - & - \\
\hline Jania corniculata & - & - & - & - & 1 & - & - & - & - \\
\hline Jania rubens & - & - & - & - & + & - & - & - & - \\
\hline Leptopsammia pruvotii & - & + & - & - & - & - & - & - & - \\
\hline Lissoclinum perforatum & - & - & - & - & - & - & - & - & - \\
\hline Lomentaria ercegovicii & - & - & - & - & - & - & + & - & - \\
\hline Obelia bidentata & - & - & - & - & - & - & + & - & - \\
\hline Pleraplysilla spinifera & - & - & - & - & - & - & 1 & - & - \\
\hline Polysyncraton sp. & - & - & - & - & - & - & - & - & - \\
\hline Porella cervicornis & - & 1 & - & - & - & + & - & - & - \\
\hline Reptadeonella violacea & - & - & - & - & - & - & - & - & - \\
\hline Rynchozoon sp II. & - & - & - & - & - & + & - & - & - \\
\hline Savygniella lafontii & - & 2 & - & - & - & - & - & - & - \\
\hline Turbicellepora magnicostata & - & - & - & - & - & + & - & - & - \\
\hline Valonia macrophysa & - & - & - & - & - & - & - & - & - \\
\hline Acanthella acuta & - & - & - & - & - & - & - & - & - \\
\hline Aglaophenia acacia & - & - & - & - & - & - & + & - & - \\
\hline Aglaophenia kincherpaueri & - & - & - & - & + & - & - & - & - \\
\hline Aglaothamnion tripinnatum & - & - & - & - & - & - & + & - & - \\
\hline Alicia mirabilis & - & + & - & - & - & - & - & - & - \\
\hline Amphiroa cryptarthrodia & - & - & - & - & - & - & - & - & - \\
\hline Aplysina cavernicola & - & - & - & - & - & - & + & - & - \\
\hline Aplysilla sulphurea & - & - & - & - & - & - & - & - & - \\
\hline Bolma rugosa & - & - & - & - & - & - & - & - & - \\
\hline Caberea boryi & - & - & - & - & - & - & + & - & - \\
\hline Calliostoma zizyphinum & - & - & - & - & - & - & - & - & - \\
\hline Celleporina caminata. & - & - & - & - & - & + & - & - & - \\
\hline Centrostephanus longispinus & - & - & - & - & - & - & + & - & - \\
\hline Cereus pedunculatus & - & - & - & - & - & - & - & - & - \\
\hline Corallina granifera & - & - & - & - & - & - & - & - & - \\
\hline Cornularia cornucopiae & - & - & - & - & - & - & - & - & - \\
\hline Crella elegans & - & - & - & - & - & - & - & - & - \\
\hline Cutleria adspersa & - & - & - & - & - & - & - & - & - \\
\hline Cutleria monoica & - & - & - & - & - & - & - & - & - \\
\hline Dardanus callidus & - & - & - & - & - & - & - & - & - \\
\hline Dentiporella sardonica & - & - & - & - & - & - & - & - & - \\
\hline Dictyonella pelligera & - & - & - & - & - & - & - & - & - \\
\hline Didemnum cf. commune & - & - & - & - & - & - & - & - & - \\
\hline Didemnum coriaceum & - & - & - & - & - & + & - & - & - \\
\hline Epizoanthus arenaceus & - & 1 & - & - & - & - & - & - & - \\
\hline Eupolymnia sp. & - & - & - & - & - & - & - & - & - \\
\hline Gelidium latifolium & - & - & - & - & + & - & - & - & - \\
\hline Hacelia attenuata & - & - & - & - & - & - & - & - & - \\
\hline Haraldia lenormandii & - & - & - & - & - & - & - & - & - \\
\hline Holothuria sp. & - & - & - & - & - & - & - & - & - \\
\hline Cacospongia scalaris & - & - & - & - & - & - & - & - & - \\
\hline Kallymenia sp. & - & - & - & - & - & - & - & - & - \\
\hline Oscarella sp. & - & - & - & - & - & - & - & - & - \\
\hline Paramuricea clavata & - & - & - & - & - & - & 1 & - & - \\
\hline Parasmittina tropica & - & - & - & - & - & - & - & - & - \\
\hline Peyssonnelia magna & - & - & - & - & - & - & - & - & - \\
\hline Peyssonnelia rubra & - & - & - & - & - & - & - & - & - \\
\hline Phorbas tenacior & - & - & - & - & - & - & + & - & - \\
\hline Platoma cyclocolpa & - & - & - & - & - & - & - & - & + \\
\hline Unidentified Polychaeta & - & - & - & - & - & - & - & - & - \\
\hline Polycyathus muellerae & - & - & - & - & - & - & - & - & - \\
\hline Polysiphonia scopulorum & - & - & - & - & - & - & - & - & - \\
\hline Polysiphonia sp. & - & - & - & - & - & - & - & - & - \\
\hline Pterothamnion plumula & - & - & - & - & - & - & - & - & - \\
\hline Reniera sp. & - & - & - & - & - & - & - & - & + \\
\hline Schizomavella cuspidata & - & - & - & - & - & - & + & - & - \\
\hline Scinaia sp. & - & - & - & - & - & - & - & - & - \\
\hline Serpulorbis arenarius & - & - & - & - & - & - & - & - & - \\
\hline Stephanoscyphus mirabilis & - & - & - & - & - & - & + & - & - \\
\hline Turbicellepora plana & - & - & - & - & - & + & - & - & - \\
\hline Ulosa stuposa & - & - & - & - & - & - & - & - & - \\
\hline Veretillum cynomorium & + & - & - & - & - & - & - & - & - \\
\hline Walkeria uva & 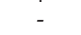 & 1 & - & - & - & - & - & - & - \\
\hline
\end{tabular}




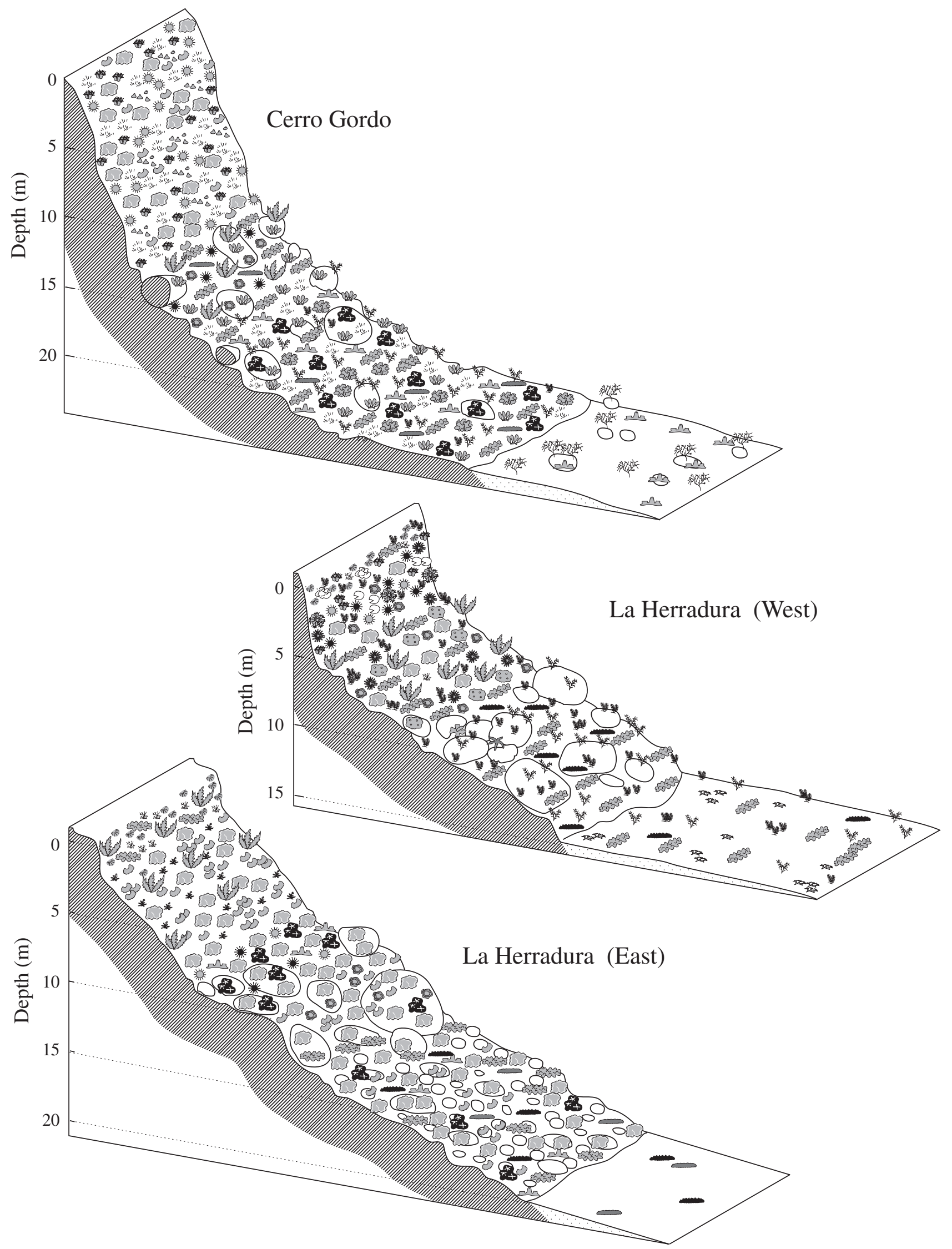

FIG. 2. - Diagrammatic representation of rocky bottom transects: Cerro Gordo (CG), La Herradura-East (HE), La Herradura-West (HW). 


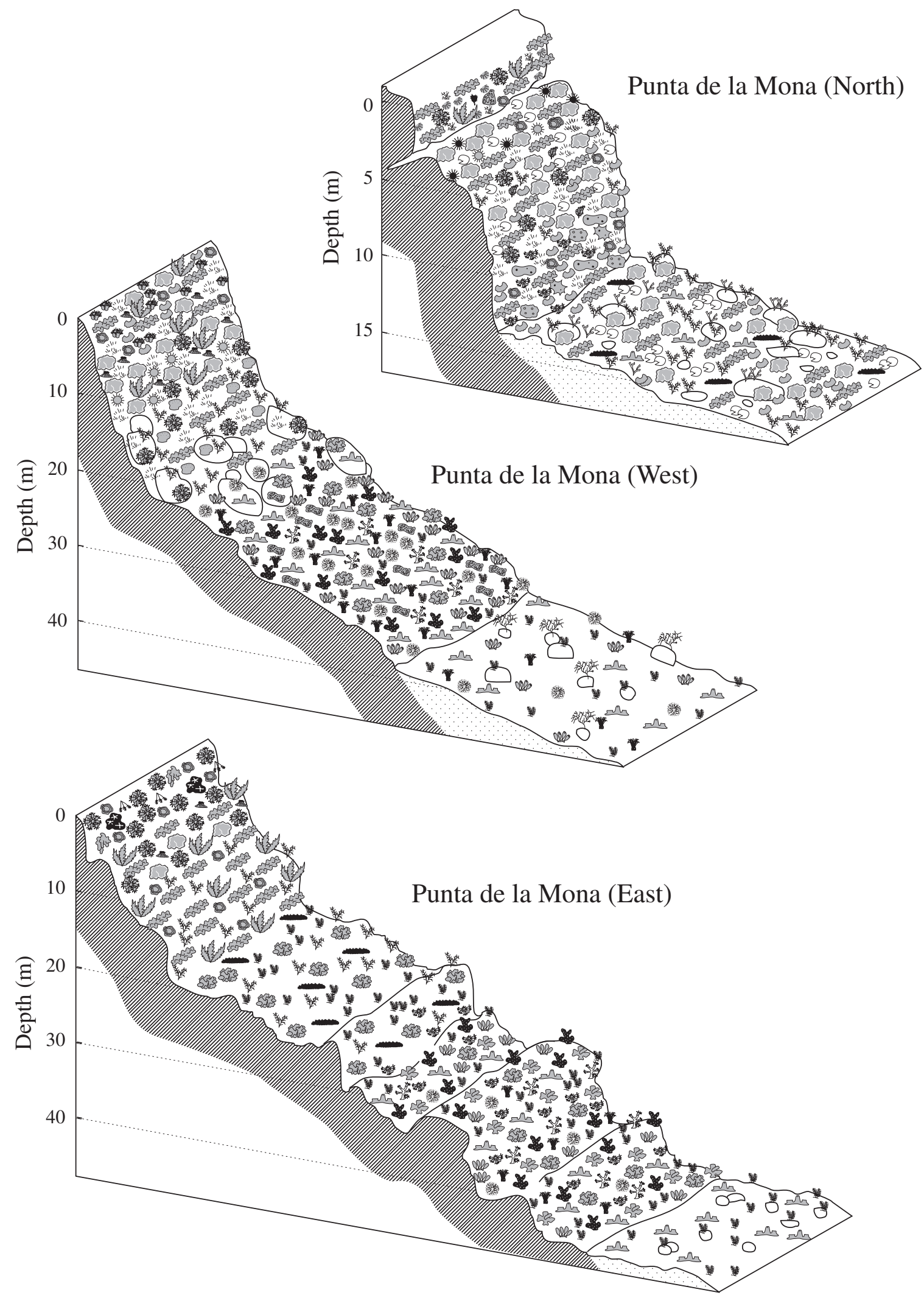

FIG. 3. - Diagrammatic representation of rocky bottom transects: Punta de la Mona-North (PN) transects, Punta de la Mona-West (PW) and Punta de la Mona-East (PE) . 


\section{Dominant species}

\begin{tabular}{|c|c|c|c|c|c|}
\hline 舴 & Bryopsis plumosa & 203 & Axinella damicornis & 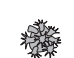 & Astroides calycularis \\
\hline 龇 & Halopteris filicina & $\odot$ & Chondrosia reniformis & 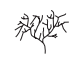 & Leptogorgia sarmentosa \\
\hline ○ & Aglaozonia sp. & $\Omega$ & Cliona viridis & 㺟然 & Parazoanthus axinellae \\
\hline R & Dictyota dichotoma & $\Delta \Delta_{\Delta}$ & Cliona celata & $x$ & Alcyonium acaule \\
\hline \multirow{2}{*}{ 运3 } & Colpomenia sinuosa & $\&$ & Crambe crambe & 盟 & Alcyonium palmatum \\
\hline & Asparagopsis armata & 60 & Clathrina coriacea & 啮 & Aglaophenia sp. \\
\hline 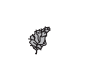 & Plocamium cartilagineum & $\ddot{\eta r}$ & Dysidea avara & 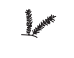 & Nemertesia antennina \\
\hline 果 & Corallina elongata & $\because:$ & Ircinia fasciculata & 筷 & Dendrophyllia ramea \\
\hline 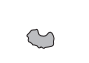 & Peyssonnelia rosa-marina & \{\}$_{3}$ & Oscarella lobularis & 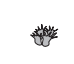 & Actinothoe sphyrodeta \\
\hline$\omega$ & Lithophyllum incrustans & & & 楼 & Aiptasia mutabilis \\
\hline 楼 & Amphiroa beauvoisii & 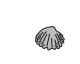 & Acanthocardia tuberculata & 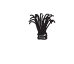 & Cerianthus membranaceus \\
\hline$\varepsilon_{0} \approx$ & Mesophyllum alternans & 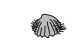 & Acanthocardia aculeata & m & Actinia sp. \\
\hline 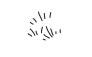 & Filamentous red algae & 最 & Aporrhais pespelecani & 警 & Eudendrium spp. \\
\hline \multirow[t]{2}{*}{ * } & Gelidium pusillum & $\infty$ & Cerithium vulgatum & 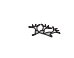 & Clavularia crassa \\
\hline & & क्री & Dardanus arrossor & & \\
\hline\{\} & Serpulidae & a & Clibanarius erythropus & 0 & Schizobrachiella sanguinea \\
\hline 䊁 & Salmacina dysteri & & & $\forall$ & Chartella tenella \\
\hline \multirow[t]{2}{*}{ y } & Phyllochaetopterus socialis & 婆 & Paracentrotus lividus & हाइ & Pentapora fascialis \\
\hline & & 涉 & Arbacia lixula & wate & Bugula neritina \\
\hline O & Pseudodistoma crucigaster & m & Holothuria tubulosa & \& & Amathia semiconvoluta \\
\hline , & Didemnum maculosum & $\infty$ & Holothuria sanctori & & \\
\hline Des & Clavelina dellavallei & is & Marthasterias glacialis & & \\
\hline
\end{tabular}

FIG. 4. - Legend for species-diagrams shown in Figure 3 and 4.

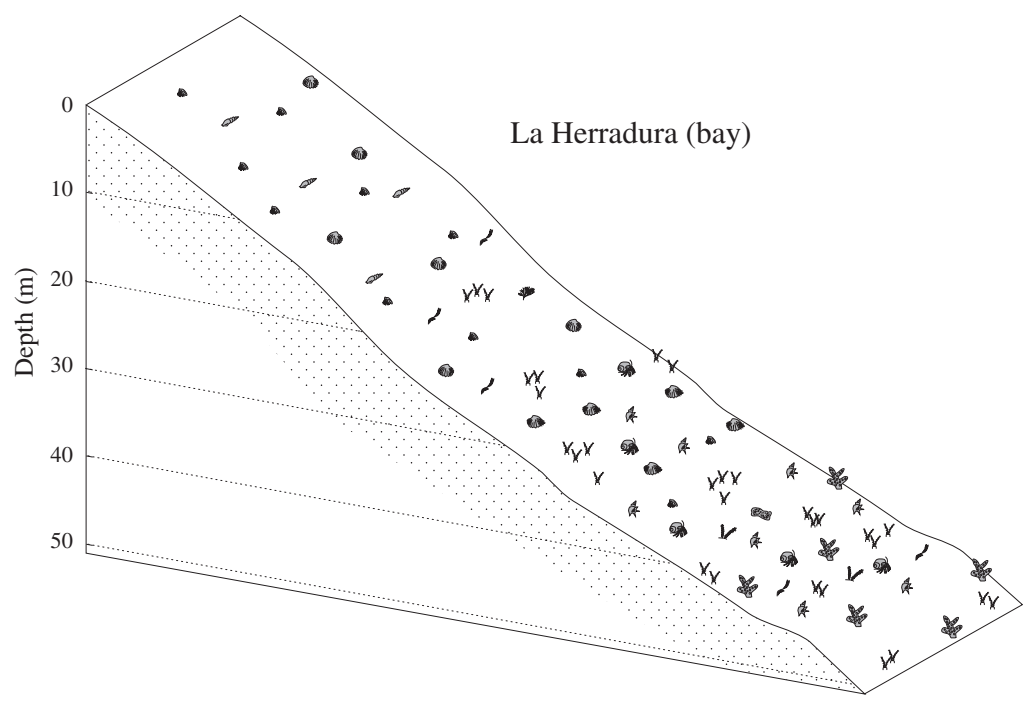

FIG. 5. - Zonation pattern of soft bottom benthic communities at La Herradura bay. 


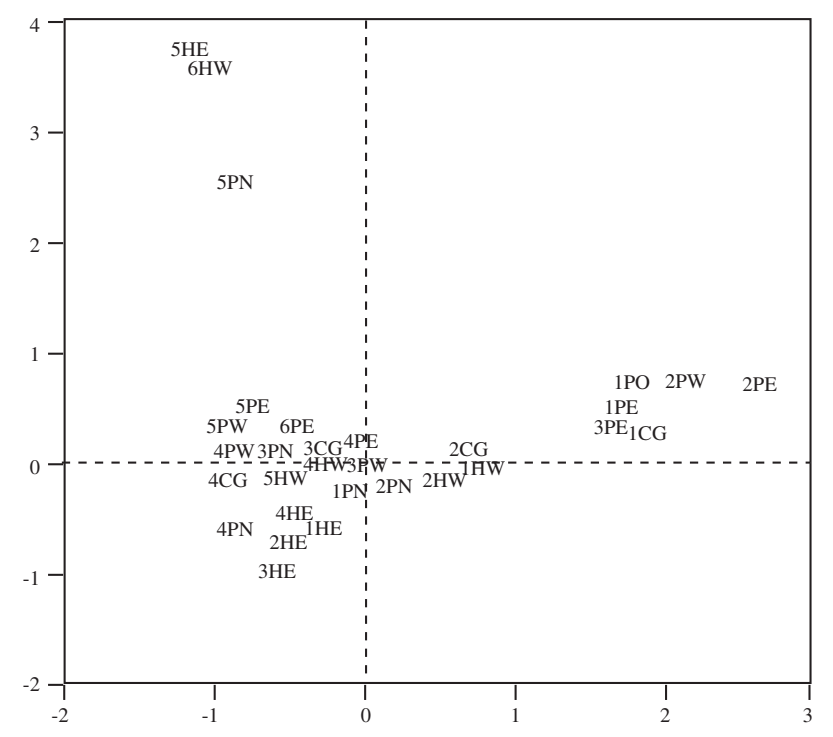

FIG. 6. - Sample representation in the two first axes derived from a factorial correspondence analysis. Sample codes correspond to those appearing in Table 1.

\section{Rocky bottoms (Figs. 2, 3)}

Ordination of samples along the two first axes discriminated by the factorial correspondence analysis shows the presence of three clusters (Fig. 6). The first axis discriminates between samples collected below 25 meters depth and those collected above, whilst the second axis segregates the samples collected above $5 \mathrm{~m}$ depth from all the rest. Samples collected between 5 and $25 \mathrm{~m}$ depth are in the same big cluster near the origin, but within this group the deepest communities are situated at positive values of axis I, whilst the shallowest samples are situated at negative values of axis I. The cumulative percentage of variance explained by the species data for the first two axes was only $28.2 \%$ ( $15.7 \%$ axis I and $12.5 \%$ axis II), as it corresponds to a highly variable data set.

Shallow water samples are characterised by two geniculate coralline algae which are very common above $5 \mathrm{~m}$ depth (Amphiroa beauvoisii and Corallina elongata), and also by the corallimorph Corynactis viridis (Fig. 7). The encrusting coralline algae, Mesophyllum alternans and Lithophyllum incrustans, although not restricted to these depths, are the most abundant species for all transects, whilst Peyssonnelia rosa marina is especially found in sheltered conditions. Red algal turfs are regularly present. Asparagopsis armata is the most abundant soft erect alga. Suspension feeders are also present and even abundant in very shallow waters; besides the scleractinian Astroides calycularis, other common species include the sponge Clathrina cerebrum, the sea anemone Actinothoe sphyrodeta, and the bryozoan Schizobrachiella sanguinea. Sea urchins Paracentrotus lividus and Arbacia lixula are sometimes abundant but they are absent in the surf zone (Figs. 2, 3).

Samples thriving at intermediate depths (5 to 25 $\mathrm{m})$ are characterised by most of the recorded algae and sea urchins (Fig. 7). There are species that are more abundant in shallow waters (algae: Asparagopsis armata, both in the gametophytic and the tetrasporophytic phase of Falkenbergia rufolanosa, Lithophyllum incrustans, Codium bursa, Aglaozonia sp.; sponges: Cliona celata, Clathrina cerebrum; cnidarians: Actinia sp., Actinothoe sphyrodeta, Astroides calycularis; sea urchins: Arbacia lixula, Paracentrotus lividus, Sphaerechinus granularis) that are situated at negative values of the axis I (Fig. 7 ), and species that prefer deeper waters (algae: Rhodymenia ardissonei, Halopteris filicina, Peyssonnelia spp.; sponges: Dysidea avara, Ircinia fasciculata, Axinella damicornis, Chondrosia reniformis; cnidarians: Parazoanthus axinellae, Aglaophenia spp.; bryozoans: Myriapora truncata and Pentapora fascialis; sea cucumbers: Holothuria tubulosa) that are mostly situated at positive values of the axis I (Fig. 7). Other species are common everywhere, like the sponges Crambe crambe and Cliona viridis, and the bryozoan Schizobrachiella sanguinea. Relative abundances of suspension feeders increase with depth and they are more abundant

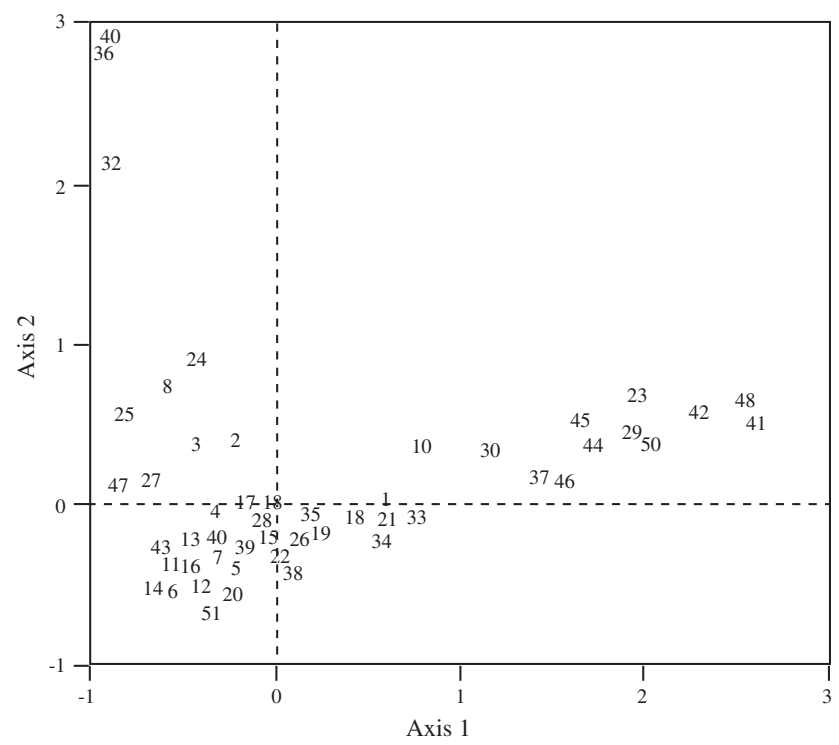

FIG. 7. - Species representation in the two first axes derived from a factorial correspondence analysis. Numbers correspond to species as indicated in Table 2. 
TABLE 3. - Species composition and abundance in the communities distinguished along the sedimentary transect, according to Braun Blanquet index. Key signs as in Table 2.

\begin{tabular}{|c|c|c|c|c|c|}
\hline & $-10 \mathrm{~m}$ & $-20 m$ & $-30 m$ & $-40 m$ & $-50 \mathrm{~m}$ \\
\hline Acanthocardia aculeata & - & 2 & 3 & + & - \\
\hline Aglaophenia sp. & - & - & - & 1 & - \\
\hline Aglaothamnion sp. & - & + & - & - & - \\
\hline Alcyonium palmatum & - & + & - & 2 & 2 \\
\hline Amathia semiconvoluta & + & 2 & 2 & 2 & - \\
\hline Unidentified Actinaria & - & - & 1 & - & - \\
\hline Anomia ephippium & - & 1 & 2 & - & - \\
\hline Antedon mediterranea & - & + & - & - & - \\
\hline Aphanocladia stichidiosa & - & + & - & - & - \\
\hline Aporrhais pespelecani & - & + & 2 & 2 & - \\
\hline Balanus sp. & - & - & - & 1 & 1 \\
\hline Bothus podas & + & - & - & - & - \\
\hline Botryllus sp. & - & 1 & - & - & - \\
\hline Buccinulum corneum & - & + & + & - & - \\
\hline Bugula neritina & - & 2 & 1 & 1 & - \\
\hline Callista chione & 1 & 1 & 1 & - & - \\
\hline Cancellaria cancellata & + & + & + & - & - \\
\hline Ceramium diaphanum & - & + & - & - & - \\
\hline Cerithium vulgatum & + & - & - & - & - \\
\hline Cladophora sp. & - & + & - & - & - \\
\hline Clibanarius erythropus & 3 & 1 & 2 & - & - \\
\hline Chamelea gallina & 3 & 1 & + & - & - \\
\hline Dardanus arrossor & - & 1 & + & 1 & - \\
\hline Didemnum maculosum & - & + & - & 2 & - \\
\hline Donacilla cornea & + & - & - & - & - \\
\hline Donax sp. & 2 & 1 & - & - & - \\
\hline Epizoanthus arenaceus & - & 1 & 2 & 1 & - \\
\hline Eunicella verrucosa & - & - & - & + & - \\
\hline Falkenbergia rufolanosa & - & + & - & - & - \\
\hline Glycymeris sp. & - & 1 & + & - & - \\
\hline Gracilaria sp. & - & + & - & - & - \\
\hline Griffithsia sp. & - & + & - & - & - \\
\hline Nassarius denticulata & - & + & + & - & - \\
\hline Nassarius reticulata & + & - & + & - & - \\
\hline Laevicardium oblongum & - & - & + & - & - \\
\hline Lepas sp. & - & - & - & + & - \\
\hline Liocarcinus vernalis & - & + & - & - & - \\
\hline Lithophyllum incrustans & - & + & - & - & - \\
\hline Lomentaria ercegovicii & - & + & - & - & - \\
\hline Lunatia sp. & - & + & - & - & - \\
\hline Mesophyllum alternans & - & 1 & 1 & - & - \\
\hline Myxicola infundibulum & - & 1 & + & - & - \\
\hline Nemertesia antennina & - & - & - & 2 & - \\
\hline Anotrichium furcellatum & - & + & - & - & - \\
\hline Obelia bidentata & - & - & - & - & 1 \\
\hline Pagurus anachoretus & - & + & - & - & - \\
\hline Paraerythropodium coralloid & des- & + & - & + & - \\
\hline Phyllochaetopterus socialis & 2 & 2 & - & 3 & 2 \\
\hline Phymanthus pulcher & - & - & + & - & - \\
\hline Pterothamnion crispum & - & + & - & - & - \\
\hline Rhodophyllis divaricata & - & - & + & - & - \\
\hline Rudicardium tuberculatum & 3 & 2 & + & - & - \\
\hline Rynchozoon sp. & - & - & + & - & - \\
\hline Salmacina dysteri & - & - & - & 1 & - \\
\hline Scalpellum scalpellum & - & - & - & + & - \\
\hline Scorpaena notata & - & - & - & 1 & - \\
\hline Scrupocellaria sp. & - & + & - & - & - \\
\hline Schizobrachiella sanguinea & - & 1 & 1 & - & - \\
\hline Sepia officinalis & + & - & - & - & - \\
\hline Serpulidae & - & + & + & + & - \\
\hline Sertularella polyzonias & - & - & - & + & 1 \\
\hline Solenidae & - & 1 & - & - & - \\
\hline Sphaeronassa mutabilis & + & - & - & - & - \\
\hline Sabella spallanzani & - & - & - & - & 1 \\
\hline Synthecium evansi & - & - & - & 1 & - \\
\hline Tellina pulchella & - & - & + & - & - \\
\hline Trachinus draco & + & - & - & - & - \\
\hline Unidentified tunicate & - & 1 & - & 1 & - \\
\hline Turritella communis & - & - & 1 & + & - \\
\hline Venus verrucosa & - & + & - & + & + \\
\hline Veretillum cynomorium & - & - & - & 1 & - \\
\hline
\end{tabular}

in crevices, boulder zones, and north-facing slopes (e.g. Punta de la Mona-North transect) (Fig. 3).

The group of samples collected in deep waters is characterised by large suspension feeders that are more or less restricted to these areas (Fig. 7): the cnidarians Dendrophyllia ramea, Leptogorgia sarmentosa, Parazoanthus axinellae, Cerianthus membranaceus and Alcyonium acaule; the sponges Axinella damicornis and Hemimycale columella; the tunicates Didemnum maculosum and Clavelina dellavallei; the bryozoan Pentapora fascialis; and the colonial worm Salmacina dysteri. Other more ubiquituous species also very common at these depths include the sponge Cliona viridis, hydroids of the genus Aglaophenia, and bryozoans such as Rynchozoon sp. and Savygniella lafontii. The detritic muddy bottoms of these deepest sites are colonised by the same suspension feeders but with a highly scattered distribution and growing on the detritic cobbles. Communities dominated by these organisms are restricted to transects of Punta de la Mona East and West (see Fig. 3).

\section{Sedimentary bottoms (Fig. 5)}

The medium grain size decreases all along the transect, with a discontinuity between 20 and $30 \mathrm{~m}$ depth, where fine sand is replaced by very fine sand and mud. (Fig. 8)

The bivalve Acanthocardia tuberculata, the gastropod Cerithium vulgatum and the hermit crab Clibanarius erythropus are abundant at $10 \mathrm{~m}$ depth. At $20 \mathrm{~m}$, the worm Phyllochaetopterus socialis is abundant inside the sediment and Acanthocardia tuberculata is also common. The bryozoans Amathia semiconvoluta and Bugula neritina colonise dead mollusc shells and small detritic stones (Fig. 5).

Between 30 and $40 \mathrm{~m}$ depth, Phyllochaetopterus socialis and Acanthocardia aculeata are the main infaunal species. Epibenthic macrofauna include the hermit crab Dardanus arrossor and the gastropod Aporrhais pespelecani. Dead mollusc shells provide a substratum for the hydroids Nemertesia antennina and Synthecium evansii and the bryozoan Amathia semiconvoluta. The soft coral Alcyonium palmatum is particularly abundant at $40 \mathrm{~m}$ depth (Fig. 5).

There is an impoverishment of the macrofauna at $50 \mathrm{~m}$ depth, where only Phyllochaetopterus socialis and Alcyonium palmatum are abundant. Below $50 \mathrm{~m}$ depth, samples collected by dredging at 60, 70, 80, 90 and $100 \mathrm{~m}$ depth show an assemblage dominated by the sea-pen Pennatula phosphorea. 


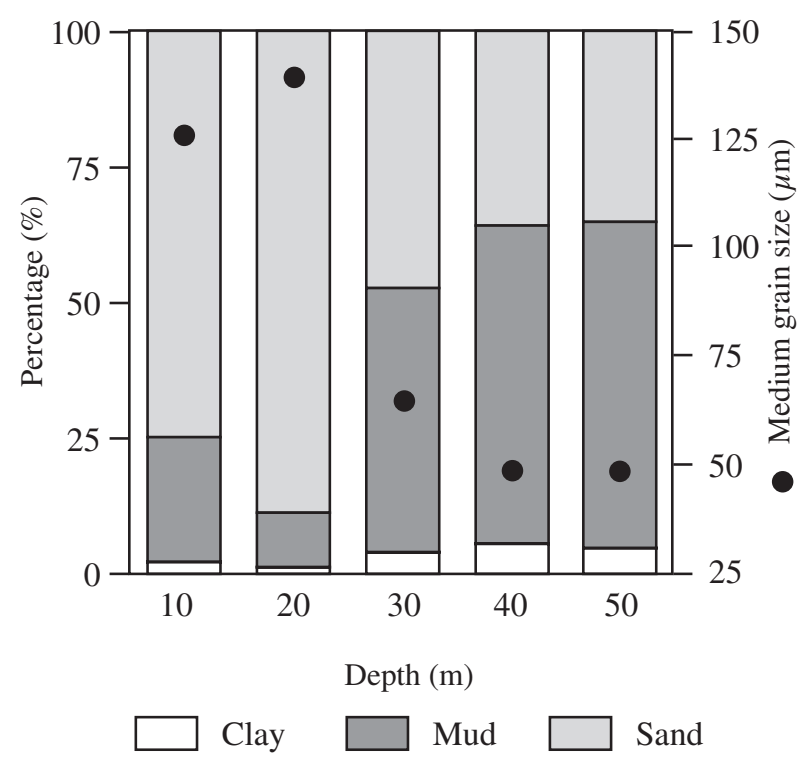

FIG. 8. - Bathymetric distribution of the different sort of sediment along the sedimentary transect.

\section{DISCUSSION}

Most of the species found in this study have a wide distribution and are common for temperate Mediterranean and Atlantic waters. According to the literature (Conde, 1989; González, 1994) most of the algal species of tropical affinities inhabiting the Mediterranean have their westernmost distribution limit at about $3^{\circ} \mathrm{W}$ longitude, and this is also the case for several Mediterranean endemisms (although other typical Mediterranean species such as Posidonia oceanica have western limits that may include La Herradura inside their distribution area; Fig. 9). Conversely, no or very few genuine Atlantic species of macrophytes are found east of about $4^{\circ} 30^{\prime} \mathrm{W}$ longitude in southern Spain (Fig. 9), so its absence in La Herradura was not unexpected. Therefore, La Herradura is situated east from the expected distribution of Atlantic species and west from most of the Mediterranean species with tropical affinities, but not from other Mediterranean species. Nevertheless, none of these last species have been found (e.g. Posidonia oceanica, Cystoseira spin$o s a$ ), with the exception of Rissoella verruculosa, a mediolittoral species that does not appear in our sublittoral samples, but which is present in La Herradura (Flores-Moya et al., 1998).

It is also interesting to note that the segregation pattern between Atlantic and Mediterranean, and subtropical to tropical species of algae is not the same on the North African coasts, where Tres Forcas Cape $\left(3^{\circ} \mathrm{W}\right)$ acts as an eastern limit for a large number of Atlantic species and a western limit for species of tropical affinities. Tres Forcas Cape (Fig. 9) is also the western limit for several Mediterranean species (González, 1994).

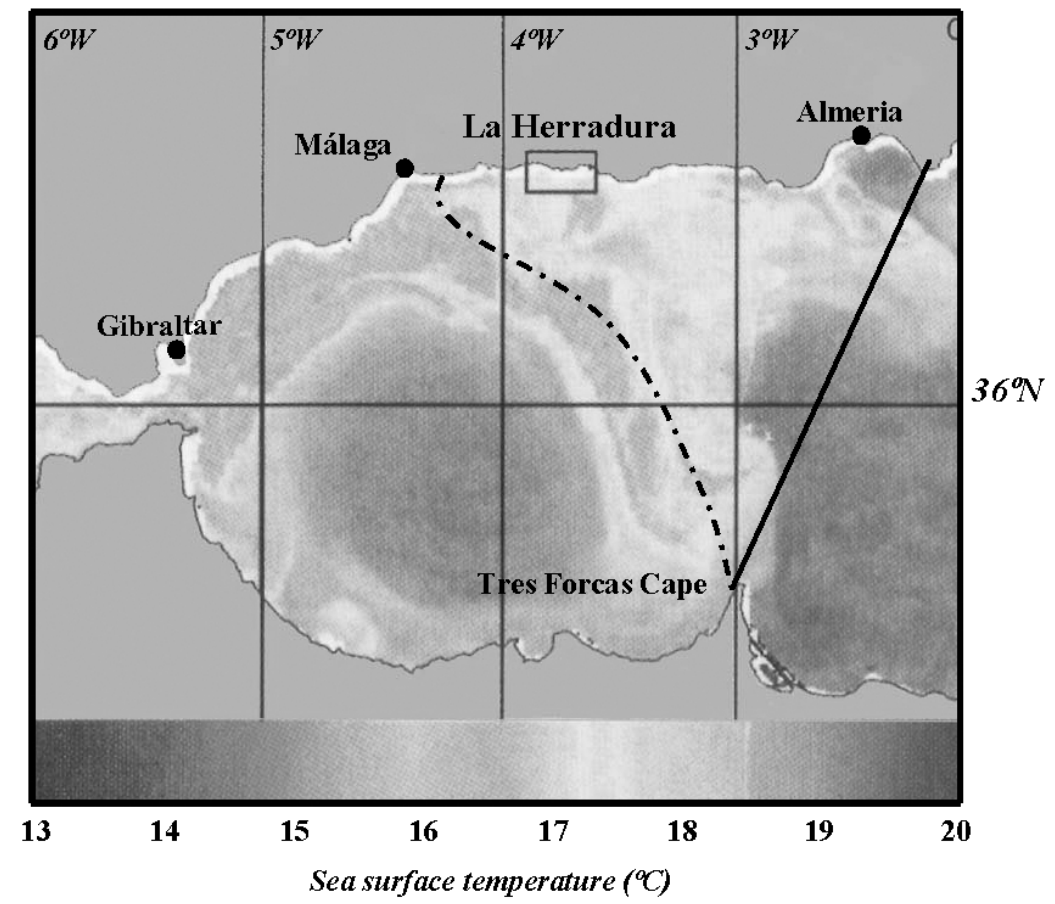

FIG. 9. - Westernmost distribution limits of most of the shallow Mediterranean sublittoral endemic algal species (e.g. Cystoseira mediterranea, $C$. crinita, $C$. ercegovicii) and tropical to subtropical algal species present in the Mediterranean (e.g. Caulerpa prolifera, Halimeda tuna, Dasycladus vermicularis, Acetabularia acetabulum) (solid line), and easternmost limit of most eu-atlantic algal species (e.g. Gelidium sesquipedale, Gigartina pistillata, Fucus spiralis) (broken line) according to data compiled by González (1994). 
The zonation pattern of La Herradura rocky bottoms greatly differs from that described for other localities in the western Mediterranean (e.g. Feldmann, 1937; Molinier, 1960; Pérès and Picard, 1964; Giaccone and Bruni, 1973; Gili and Ros, 1985; Boudouresque et al., 1986; Soto and Conde, 1989; Ballesteros and Ros, 1989; Ballesteros, 1992; García-Raso et al., 1992; Ballesteros et al., 1993; Giaccone et al., 1994; Canals and Ballesteros, 1997; Calvín, 2003). Shallow benthic communities in Mediterranean waters are usually dominated by erect macroalgae belonging to the Fucophyceae (genera Cystoseira, Padina, Halopteris, Cladostephus, Dictyota), Rhodophyceae (Liagora, Laurencia, Corallina, Amphiroa) or Chlorophyceae (Acetabularia, Dasycladus, Caulerpa), with some scattered suspension feeders (mainly sponges: genera Crambe, Ircinia, Sarcotragus) and sea-urchins (Paracentrotus lividus, Arbacia lixula). Shallow water benthic communities in nearby Atlantic areas are characterised by belts of large erect algae (genera Cystoseira, Sargassum, Saccorhiza, Halopteris, Corallina, Pterocladia, Gelidium, Halopitys and Sphaerococcus) (Seoane-Camba, 1965; OteroSchmitt and Pérez-Cirera, 2002) different to those described from the Mediterranean and different also to those described in this paper for La Herradura. La Herradura infralittoral bottoms also differ from bottoms situated near the Alboran island which are biogeographically Atlantic (Giaccone, 1972; Conde, 1989) although they are situated more than two hundred kilometers inside the Mediterranean.

Deep (20-50 m depth) Mediterranean rocky bottom communities are characterised by the dominance of algae specially adapted to dim light conditions such as the encrusting corallines Mesophyllum alternans and Lithophyllum stictaeforme, and Peyssonnelia spp., with erect algae Cystoseira zosteroides, Halopteris filicina, Flabellia petiolata and Halimeda tuna, amongst others (Feldmann, 1937; Boudouresque, 1973; Boudouresque et al., 1986; Ballesteros, 1990, 1991; Ballesteros et al., 1993). The growth, death and accretion of encrusting corallines builds-up a biogenic structure called coralligenous that is typical of Mediterranean circalittoral rocky bottoms (Ros et al., 1985). Many species of suspension feeders grow over the coralligenous banks (Ballesteros et al., 1993) and their abundance is related to depth, currents and available particulate organic matter (Zabala and Ballesteros, 1989). On the other hand, the coralligenous structure is absent in Atlantic waters near the straits of Gibraltar, and kelp beds dominated by Laminaria ochroleuca and Cystoseira usneoides develop at the 20-50 m bathymetric range (Werner, 1962). Coralligenous or kelp beds are absent in La Herradura deep waters and the floristic component is very poor and scarcely developed. The dominance corresponds to big suspension feeders such as Cerianthus membranaceus, Salmacina dysteri, Pentapora fascialis and Dendrophyllia ramea, species that are also common in both the southwestern Mediterranean and Atlantic deep waters.

Most species of fauna and flora dwelling in $\mathrm{La}$ Herradura sea bottoms have an Atlantic-Mediterranean distribution, and are common in both. Benthic communities are neither typically Atlantic nor typically Mediterranean, since most genus and species defining Atlantic or Mediterranean communities are lacking. We suggest that deep water upwellings peculiar to this area (Lanoix, 1974; Cano, 1978; Rodríguez, 1990) prevent the development of the typical Mediterranean species and communities thriving in shallow waters. However, since the deep waters are of Mediterranean origin, pure Atlantic species and communities are also absent. This situation described for La Herradura can probably be extrapolated to the coasts of southern Spain affected by the upwelling, that is between $3^{\circ}$ and $4^{\circ} 30^{\prime} \mathrm{W}$. This reasoning could also explain the biogeographical asymmetry found between southern Spain and northern Morocco. Much research is needed in this geographical area to support this assessment.

The zonation patterns of La Herradura are well defined, as can be easily observed in Figures 3 and 4 and has been demonstrated in the ordination analysis for the rocky bottoms. Both axes I and II are related to depth, a situation that is predictable when sampling has been performed along a strong environmental gradient (Legendre and Legendre, 1979; Ballesteros and Romero, 1988). The ordination of samples defined by the first and second axes highlights two discontinuities. The first, situated at around $25 \mathrm{~m}$ depth, can be interpreted as the change from algal to animal dominance and can be interpreted as the boundary between the infralittoral and the circalittoral zone. The second one is situated above $5 \mathrm{~m}$ depth and may be related to the change from highly reophilic species that withstand heavy surf and hydrodynamism (upper infralittoral zone) to species not adapted to surf or to strong water movement (lower infralittoral zone). 


\section{ACKNOWLEDGEMENTS}

This study was financially supported by the MATER project (MAST-III-CT95-0024). The authors thank Natàlia Sant and Francisco J. Viciana for their help in the field work, María Jesús Uriz, Manuel Maldonado Xavier Turón, Mikel Zabala, Joao Gil, and Rafael Sardá for the identification of some species, and Miquel Canals for logistic support. We also thank the Centro de Buceo La Herradura for providing diving facilities.

\section{REFERENCES}

Ballesteros, E. - 1990. Structure and dynamics of the community of Cystoseira zosteroides (Turner) C. Agardh (Fucales, Phaeophyceae) in the Northwestern Mediterranean. Sci. Mar., 54: 217-229.

Ballesteros, E. - 1991. Structure of a deep water community of Halimeda tuna (Chlorophyceae, Caulerpales) from the Northwestern Mediterranean. Collect. Bot., 20: 5-21.

Ballesteros, E. - 1992. Els vegetals i la zonació litoral: espècies, comunitats i factors que influeixen en la seva distribució. Arx. Secc. Ciències, 101. Institut d'Estudis Catalans, Barcelona.

Ballesteros, E. and J. Romero. - 1988. Zonation patterns in tideless environments (Northwestern Mediterranean): looking for discontinuities in species distributions. Inv. Pesq., 52(4): 595-616.

Ballesteros, E. and J. Ros. - 1989. Els ecosistemes bentònics. In: R. Folch (ed.), Història Natural dels Països Catalans, Vol. XIV. Sistemes Naturals, pp. 119-176. Enciclopèdia Catalana, Barcelona.

Ballesteros, E., M. Zabala, M.J. Uriz, A. Garcia-Rubies and X. Turon. - 1993. El bentos: les comunitats. In: J.A. Alcover, E. Ballesteros and J.J. Fornós (eds.), Història Natural de l'Arxipèlag de Cabrera. pp. 687-730. CSIC-Ed. Moll, Palma de Mallorca.

Boudouresque, C.F. - 1973. Recherches de bionomie analytique, structurale et expérimentale sur les peuplements benthiques sciaphiles de Méditerranée Occidentale (fraction algale): les peuplements sciaphiles de mode relativement calme sur substrats durs. Bull. Mus. Hist. Nat. Marseille, 33: 145-225.

Boudouresque, C.F., J.G. Harmelin and A. Jeudy de Grissac (eds.). - 1986. Le benthos marin de l'île de Zembra (Parc National, Tunisie). GIS Posidonie. Marseille.

Braun Blanquet, J. - 1979. Fitosociología. Blume, Madrid.

Calvín, J.C. - 2003. Fondos marinos de Murcia: tipos, paisajes, flora y fauna, estado de conservación y mejores inmersiones. Ed. Juan Carlos Calvín, Murcia.

Canals, M. and E. Ballesteros. - 1997. Production of carbonate particles by phytobenthic communities on the Mallorca-Menorca shelf, northwestern Mediterranean Sea. Deep Sea Res. II, 44: 611-629.

Cano, A. - 1978. Resultados de la campaña "Alborán 76". Bol. Inst. Esp. Oceanogr., 4: 3-51.

Cebrian, E., Ballesteros, E., and M. Canals. - 2000. Shallow rocky bottom benthic assemblages as calcium carbonate producers in the Alboran Sea (Southwestern Mediterranean). Oceanol. Acta, 23(3): 311- 322

Conde, F. - 1989. Ficogeografía del Mar de Alborán en el contexto del Mediterráneo occidental. Anales Jard. Bot. Madrid, 46(1): 21-26.

Feldmann, J. - 1937. Recherches sur la végétation marine de la Méditerranée. La côte des Albères. Wolf. Rouen.

Flores-Moya, A., I. Gómez, B. Vinegla, M. Altamirano, E. PérezRodríguez, C. Maestre, R.M. Caballero and F.L. Figueroa. 1998. Effects of solar radiation on the endemic red alga Rissoella verruculosa: photosynthetic performance, pigment content and the activities of enzymes related to nutrient uptake. New Phytologist, 139: 673-684.

García-Raso, E., A.A. Luque, J. Templado, C. Salas, E. Hergueta,
D. Moreno and M. Calvo. - 1992. Fauna y flora marinas del Parque Natural de Cabo de Gata-Níjar. Mateu Cromo, Madrid.

Giaccone, G. - 1972. Struttura, ecologia e corologia dei popolamenti a laminariae dello Stretto di Messina e Mare di Alboran. Mem. Biol. Mar. Ocean., 2: 37-59.

Giaccone, G. and A. Bruni. - 1973. Le Cystoseire e la vegetazione sommersa del Mediterraneo. Atti. Ist. Ven. Sci. Lett. Art., 131: 59-103.

Giaccone, G., G. Alongi, F. Pizzuto and A. Cossu. - 1994. La vegetazione marina bentonica fotofila del Mediterraneo: II. Infralitorale e Circalitorale. Proposte di aggiornamento. Boll. Acc. Gioenia Sci. Nat., 27: 111-157.

Gil, J. - 1988. Hidrografía de la plataforma continental española Mediterránea y Golfo de León. Mayo, Junio. Inf. Téc. Inst. Esp. Oceánogr., 81, 1:124.

Gili, J.M. and J. Ros. - 1985. Study and cartography of the benthic communities of Medes Islands (NE Spain). P.S.Z.N.I: Mar. Ecol., 6(3): 219-238

González, J.A. - 1994. La flora marina del litoral próximo a Melilla. Ensayos Melillenses, 2. Melilla.

González, J.A. and F. Conde. - 1993. Estudio biogeográfico de las fucales y laminariales atlánticas en el litoral mediterráneo de Marruecos. Acta Bot. Malacitana, 18: 39-44.

Lanoix, F. - 1974. Étude hydrographique et dynamique de la Mer d'Alboran. NATO. Brussels, $39 \mathrm{pp}$

Legendre, L. and P. Legendre. - 1979. Écologie numérique. I. Le traitement multiple des données écologiques. II. La structure des données écologiques. Masson, Paris.

Maldonado, M. - 1992. Demosponges of the red coral bottoms from the Alboran Sea. J. Nat. Hist., 26: 1131-1161.

Maldonado, M. - 1993. Demosponjas litorales del mar de Alborán. Faunística y biogeografía. $\mathrm{PhD}$ thesis, Universidad de Barcelona.

Maldonado, M. and M.J. Uriz - 1995. Biotic affinities in a transitional zone between the Atlantic and the Mediterranean: a biogeographical approach based on sponges. J. Biogeogr., 22: 89-110.

Minas, H.J., B. Coste, P. Le-Corre, M. Minas, and P. Raimbault. 1991. Biological and geochemical signatures associate with the water circulation through the Strait of Gibraltar and the Western Alboran sea. J. Geophys. Res., 96: 8755-8771.

Molinier, R. - 1960. Étude des biocoenoses marines du Cap Corse. Vegetatio, 9: 120-192, 217-311.

Ocaña, A., L. Sánchez, S. López and J.F. Viciana. - 2000. Guía submarina de invertebrados no artrópodos. Ed. Comares, Granada.

Otero-Schmitt, J. and J.L. Pérez-Cirera. - 2002. Infralittoral benthic biocoenoses from northern Ría de Muros, Atlantic coast of northwest Spain. Botanica Marina, 45: 93-122.

Parrilla, G. and T.H. Kinder. - 1987. Oceanografía física del mar de Alborán. Bol. Inst. Esp. Oceanogr., 4: 133-165.

Pérès, J.M. - 1985. History of the Mediterranean biota and the colonization of the depths. In: R. Margalef (ed.), Western Mediterranean, pp.198-232. Pergamon, Oxford.

Pérès, J.M. and J. Picard. - 1964. Nouveau Manuel de bionomie benthique de la Mer Méditerranée. Rec. Trav. Stat. Mar. Endoume, 32(47): 1-247.

Rodríguez, J.M. - 1990. Contribución al conocimiento del ictioplancton del mar de Alborán. Bol. Inst. Esp. Oceanogr., 6: 1-20.

Ros, J., J. Romero, E. Ballesteros and J.M. Gili. - 1985. Diving in blue water: the benthos. In: R. Margalef (ed.), Western Mediterranean, pp. 233-295. Pergamon, Oxford.

Seoane-Camba, J. - 1965. Estudios sobre las algas bentónicas en la costa sur de la Península Ibérica. Inv. Pesq., 29: 3-216.

Soto, J. and F. Conde. - 1989. La zonación algal en el litoral del SE de la Península Ibérica. Fol. Bot. Misc., 6: 71-79.

Templado, J.M. García-Carrascosa, L. Baratech, R. Capaccioni, A Juan, A. López-Ibor, R. Silvestre and C. Massó. - 1986. Estudio preliminar de la fauna asociada a los fondos coralíferos del mar de Alborán (SE de España). Bol. Inst. Esp. Oceanogr., 3: 93-104.

Templado, J.M., A. Guerra, J. Bedoya, D. Moreno, J.M. Remón, M. Maldonado and M.A. Ramos. - 1993. Fauna marina circalitoral del sur de la Península Ibérica. CSIC, Madrid.

Ter Braak, C.J.F. - 1987. Ordination. In: R.H.G. Jongman, C.J.F. Ter Braak and O.F.N. Van Togenen (eds.) Data Analysis in community and lanscape ecology. pp. 91-173. Pudoc, Wageningen.

Tintoré, J., D. Gomis and S. Alonso. - 1991. Mesoscale dynamics 
and vertical motion in the Alboran Sea. J. Phys. Oceanogr., 21: 811-823.

Wentworth, W. - 1972. A scale of grade and class terms for clastic sediments. J. Geology, 30: 377-392.

Werner, R.G. - 1962. Essai d'une étude de la répartition des cryptogames marines et maritimes du Maroc. Bull. Soc. Sci. Nat. Maroc, 42: 1-33.

Zabala, M., I. Olivella, J.M. Gili and J. Ros. - 1982. Un intento de tipificación metodológica en el estudio del bentos marino accesible en escafandra autónoma. Actas Primer Simp. Ibér. Bentos Mar., 2: 961-982.

Zabala, M. and E. Ballesteros. - 1989. Surface-dependent strategies and energy flux in benthic marine communities or, why corals do not exist in the Mediterranean. Sci. Mar., 53: 1-15.

Scient. ed.: P. Abelló 